\title{
Verfahrenswahl bei Risiko
}
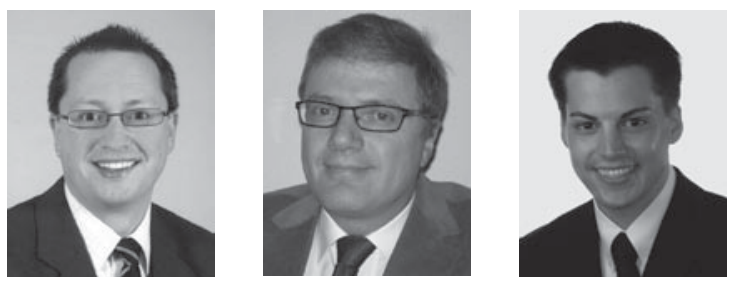

\section{Oliver Michael Dürr, Robert F. Göx, Uwe Heller}

\section{Überblick}

- Dieser Beitrag untersucht das Verfahrenswahlproblem bei Risiko. Wir zeigen, dass die unter Sicherheit abgeleiteten Entscheidungsregeln für die Verfahrenswahl bei unsicherer Leistungsmenge nur dann ihre Gültigkeit behalten, wenn ein risikoneutraler Entscheider unterstellt wird.

- Bei Risikoaversion bestimmt sich die Lösung des Verfahrenswahlproblems durch die Zielgrösse des Entscheiders. Bei kostenbasierter Betrachtungsweise erfolgt der Übergang auf Verfahren mit höheren Fixkosten und geringeren variablen Stückkosten früher als bei Sicherheit, bei deckungsbeitragsorientierter Betrachtungsweise später.

- Verfahren, die unter Risikoneutralität effizient sind, können bei Risikoaversion von anderen Verfahren dominiert werden und umgekehrt.

- Im Rahmen einer Agency-Beziehung können Verfahrenswahlprobleme trotz Risikoneutralität des Prinzipals nicht unabhängig von der Lösung des Anreizproblems gelöst werden.

- Die Resultate zeigen, dass die optimale Lösung des Verfahrenswahlproblems bei Risiko mehr als einen Kostenvergleich erfordert. Insbesondere müssen die Risikoneigung des Entscheiders und der mit dem Einsatz des Verfahrens verbundene Nutzen korrekt berücksichtigt werden.

Keywords Decision making under risk - choice among cost structures · break-even analysis

Eingegangen: 27. August 2007

JEL-Codes: D81, L23, M40

Dipl.-Oec. Oliver Michael Dürr $(\bowtie)$

Lehrstuhl für Unternehmensrechnung und Controlling, Universität Fribourg, Bd. de Pérolles 90, CH-1700 Fribourg (Schweiz), Tel.: +41 26300 8313, Fax +4126300 9659, email: olivermichael.duerr@unifr. ch.

Prof. Dr. Robert F. Göx $(\bowtie)$

Lehrstuhl für Unternehmensrechnung und Controlling, Universität Fribourg, Bd. de Pérolles 90,

CH-1700 Fribourg (Schweiz), Tel.: +41 26300 8310/8311, Fax +41 26300 9659, email: robert.goex@unifr.ch, web: http://www.unifr.ch/controlling/

Dipl.-Kfm. Uwe Heller ( $\bowtie)$

Lehrstuhl für Unternehmensrechnung und Controlling, Universität Fribourg, Bd. de Pérolles 90,

CH-1700 Fribourg (Schweiz), Tel.: +41 26300 8316, Fax +4126300 9659, email: uwe.heller@unifr.ch. 


\section{A. Einleitung}

Entscheidungen über die Wahl der kostengünstigsten Alternative aus einer gegebenen Menge von Produktionsverfahren, die sich hinsichtlich der mit ihnen verbundenen fixen und variablen Kosten unterscheiden, werden gemeinhin als Verfahrenswahlproblem oder auch als Fixkostensubstitutionsproblem bezeichnet. ${ }^{1}$ Sie gehören zu den häufigsten praktischen betriebswirtschaftlichen Fragestellungen und treten nicht nur im Produktionsbereich auf, wo etwa über den Einsatz von Maschinen mit unterschiedlichen Automatisierungsgraden, Abschreibungen und Wartungskosten entschieden werden muss, sondern vor allem auch im Bereich der Dienstleistungen. Vereinfachend lässt sich auch die Entscheidung über die Eigenfertigung oder den Fremdbezug von Bauteilen als Verfahrenswahlproblem interpretieren. ${ }^{2}$

Konkrete praktische Beispiele für Verfahrenswahlprobleme finden sich vor allem im Dienstleistungsbereich, wo häufig die Auswahl zwischen Tarifen mit unterschiedlichen Grundbeiträgen und variablen Leistungsentgelten besteht. So bieten etwa Mobilfunkanbieter die Auswahl zwischen Tarifen mit unterschiedlichen monatlichen Grundgebühren und Verbindungsentgelten pro Minute. Im Extremfall werden auch sogenannte FlatrateTarife angeboten, bei denen die Verbindungsentgelte ganz entfallen. Ein anderes Beispiel ist die Bahncard, bei der man in Abhängigkeit von der gewählten Variante gegen Zahlung eines jeweils höheren Basispreises $25 \%, 50 \%$ oder gar $100 \%$ der regulär zu zahlenden Kosten für Bahnfahrten sparen kann. Schließlich können in der Schweiz die Versicherten in der gesetzlich geregelten Grundversicherung zwischen Selbstbehalten in Höhe von 300 bis zu 2500 Franken wählen und müssen dafür im Gegenzug eine geringere monatliche Versicherungsprämie zahlen. Die genannten Beispiele repräsentieren nur eine kleine Auswahl der praktisch existierenden Verfahrenswahlprobleme und belegen die praktische Relevanz des betrachteten Problems.

Bei bekannter Bezugsgröße für die Verursachung der variablen Kosten (im Folgenden vereinfachend als Leistungsmenge bezeichnet) lassen sich Verfahrenswahlprobleme durch einen einfachen Kostenvergleich lösen. Gewählt wird das Verfahren, das für die gegebene Leistungsmenge die geringsten Gesamtkosten aufweist. Entsprechend lässt sich für beliebige Leistungsmengen jeweils die kostengünstigste Alternative angeben, wobei Verfahren mit höheren Fixkosten und geringeren variablen Kosten mit zunehmender Leistungsmenge attraktiver werden. Auch kann es vorkommen, dass einzelne Verfahren für jede Leistungsmenge von mindestens einem anderen Verfahren dominiert werden, so dass sie unter Sicherheit niemals als kostengünstigste Alternative in Betracht kommen.

Auch wenn es Situationen geben mag, in denen man vereinfachend von sicheren Erwartungen ausgehen kann, dürfte der für die Praxis weitaus relevantere Fall derjenige sein, bei dem die Leistungsmenge zum Zeitpunkt der Verfahrenswahlentscheidung nicht genau bekannt ist. Dieser Fall ist Gegenstand dieses Beitrages, in dem wir untersuchen wollen, wie sich Unsicherheit über die Leistungsmenge und die Risikoneigung des Entscheiders auf die Lösung des Verfahrenswahlproblems auswirken.

Unsere Analyse zeigt folgende Ergebnisse: Bei unsicherer Leistungsmenge und Risikoneutralität behalten die unter Sicherheit abgeleitete Entscheidungsregeln für die Verfahrenswahl grundsätzlich ihre Gültigkeit. An die Stelle der üblichen Break-Even-Bedingung für 
die bekannte Absatzmenge tritt jedoch eine entsprechende Bedingung für die erwartete Absatzmenge.

Abweichungen von den bekannten Entscheidungsregeln ergeben sich jedoch, wenn ein risikoaverser Entscheider unterstellt wird. Bei einem rein kostenbasierten Verfahrensvergleich bewirkt die Risikoaversion zunächst einen früheren Übergang auf Verfahren mit höheren Fixkosten und geringeren variablen Stückkosten als dies bei Sicherheit bzw. Risikoneutralität der Fall wäre. Dieses Resultat beruht auf der Tatsache, dass die für die Bestimmung der Risikoprämie maßgebliche Kostenvarianz überproportional in den variablen Stückkosten steigt und damit Verfahren mit hohen Fixkosten und geringen variablen Stückkosten gegenüber Verfahren mit einer gegenläufigen Struktur begünstigt.

Gemäß unserer Analyse kann dieser Effekt überdies dazu führen, dass Verfahren, die unter Risikoneutralität Element der effizienten Alternativenmenge sind, bei Risikoaversion dominiert werden. Das ist immer dann der Fall, wenn beim Verfahrensübergang der Rückgang der Risikoprämie den Zuwachs der Fixkosten übersteigt. Im Gegensatz dazu ist es aber ebenso möglich, dass Verfahren die unter Risikoneutralität von einer Kombination anderer Verfahren dominiert wurden, bei Risikoaversion zur effizienten Alternativenmenge zählen.

In einer Erweiterung unseres Grundmodells untersuchen wir, ob die aus dem kostenbasierten Verfahrensvergleich abgeleiteten Ergebnisse bei einer deckungsbeitragsorientierten Betrachtungsweise ihre Gültigkeit behalten. Dabei stellen wir jedoch fest, dass risikoaverse Entscheider erst bei einer größeren erwarteten Menge auf ein Verfahren mit geringeren variablen Stückkosten übergehen als bei Risikoneutralität. Die abweichenden Resultate bei deckungsbeitrags- und kostenorientierter Betrachtungsweise lassen sich mit der unterschiedlichen Bewertung des Risikos von Absatzmengenschwankungen erklären. Im Gegensatz zur Kostenvarianz steigt das Deckungsbeitragsrisiko mit der Abnahme der variablen Stückkosten an, so dass sich beim Übergang auf ein Verfahren mit geringeren variablen Stückkosten nicht nur die Fixkosten erhöhen, sondern auch die Risikoprämie ansteigt.

Wenn der Nutzen des Verfahrenseinsatzes bekannt ist und mit der Einsatzmenge variiert, führt damit bei Risikoaversion nur eine deckungsbeitragsorientierte Betrachtungsweise zu einer korrekten Verfahrenswahlentscheidung. Eine Verfahrenswahlentscheidung auf Basis einer isolierten Betrachtung des Kostenrisikos führt nur dann zum richtigen Ergebnis, wenn der Nutzen unabhängig von der Leistungsmenge und damit sicher ist.

Schließlich betrachten wir in einer zweiten Erweiterung des Grundmodells das Verfahrenswahlproblem im Kontext eines einfachen Prinzipal-Agenten-Modells bei dem der Prinzipal die Verfahrenswahlentscheidung trifft und der Agent mit seinem Arbeitseinsatz die Absatzmenge erhöhen kann. Im Ergebnis zeigt sich, dass die Lösung des Verfahrenswahlproblems trotz Risikoneutralität des Prinzipals vom Standardfall abweicht. Konkret macht der Beitrag des Agenten Verfahren mit höheren Deckungsbeiträgen tendenziell attraktiver, so dass der Übergang auf Verfahren mit geringeren variablen Stückkosten früher erfolgt als im Standardfall.

Unser Beitrag knüpft an die Literatur zur stochastischen Break-Even-Analyse an. ${ }^{3}$ Ein ausführlicher Überblick dazu findet sich bei Schweitzer und Troßmann (1998), die zwei Untersuchungsrichtungen unterscheiden. Zum einen geht es um Wahrscheinlichkeitsaussagen für das Erreichen von Break-Even-Punkten, und zum anderen um die Ermittlung von 
Wahrscheinlichkeiten für die Lage von Break-Even-Punkten. Keiner der bisherigen Beiträge befasst sich jedoch im Detail mit dem Verfahrenswahlproblem bei Risiko. Auch die Frage nach der geeigneten Zielgröße für die Lösung des Verfahrenswahlproblems bei Risikoaversion wurde bisher ebenso wenig untersucht, wie das Verfahrenswahlproblem im Kontext eines Agency-Modells.

Das Problem geeigneter Zielgrößen für risikoaverse Entscheider wurde in anderem Zusammenhang von Dillon and Nash (1978) untersucht. Unter Ausnutzung der Tatsache, dass der Erwartungsnutzen eines risikoaversen Entscheiders von der Höhe seines Anfangsvermögens abhängt, zeigen die Autoren auf, dass auch Vermögenspositionen, die für alle Entscheidungsalternativen identisch sind, wie etwa Fixkosten oder Gewinne anderer Bereiche, bei Risikoaversion grundsätzlich entscheidungsrelevant sind. ${ }^{4} \mathrm{Da}$ Fixkosten für Verfahrenswahlprobleme per Definition bereits bei Sicherheit relevant sind, ist dieser Effekt für unsere Analyse jedoch von untergeordneter Bedeutung. Aus Gründen der Übersichtlichkeit abstrahieren wir daher von Vermögenseffekten und unterstellen einen Entscheider mit konstanter absoluter Risikoaversion. ${ }^{5}$ Der Vollständigkeit halber zeigen wir im Anhang auf, dass die wesentlichen Ergebnisse unserer Analyse auch dann erhalten bleiben, wenn wir diese vereinfachende Annahme aufheben.

Die weitere Untersuchung ist wie folgt aufgebaut: In Abschnitt B werden das Verfahrenswahlproblem und die Präferenzen des Entscheiders spezifiziert. In Abschnitt $\mathrm{C}$ wird das Verfahrenswahlproblem bei Risiko betrachtet. Dabei untersuchen wir zunächst den Fall der Risikoneutralität und anschließend den Fall der Risikoaversion. In Abschnitt D untersuchen wir zwei Erweiterungen des Grundmodells. Zum einen erweitern wir die kostenbasierte Analyse aus Abschnitt $\mathrm{C}$ um eine Nutzenkomponente und untersuchen, unter welchen Bedingungen die Kosten- und Nutzenbetrachtungen separiert werden können. Zum anderen stellen wir das Verfahrenswahlproblem in den Kontext eines linearen Agency-Modells, bei dem der risikoneutrale Prinzipal vor der Lösung des eigentlichen Anreizproblems eine Verfahrenswahlentscheidung treffen muss. Abschnitt E beschließt diesen Beitrag mit einer kurzen Zusammenfassung.

\section{B. Modellrahmen}

Betrachtet wird die Situation eines Entscheiders, der vor dem Problem steht, sich für eines von $n$ verschiedenen Verfahren zu entscheiden, die sich hinsichtlich der Höhe ihrer fixen und variablen Kostenkomponenten voneinander unterscheiden. Die Kostenfunktion von Verfahren $i$ in Abhängigkeit von der Leistungsmenge $x$ sei wie folgt gegeben:

$$
K_{i}(x)=F_{i}+c_{i} \cdot x, \quad i=1, \ldots, n
$$

Darin bezeichnet $F_{i}$ die Fixkosten und $c_{i}$ die variablen Stückkosten des Verfahrens $i$. Die Verfahren seien eindeutig nach aufsteigender Höhe ihrer fixen Kosten geordnet, so dass $F_{1} \geq 0$ und $F_{1}<F_{2}<\ldots<F_{n}$. Zugleich gelte für die variablen Stückkosten der Verfahren eine absteigende Ordnung, so dass $c_{1}>c_{2}>\ldots>c_{n} \geq 0$. Die resultierende Folge von Verfahren mit steigenden Fixkosten und fallenden variablen Stückkosten stellt sicher, dass keines der Verfahren direkt von einem anderen Verfahren für alle möglichen Leis- 
tungsmengen dominiert wird und schließt damit triviale Lösungen des Verfahrenswahlproblems aus. ${ }^{6}$

Die Leistungsmenge $x$ sei zum Zeitpunkt der Verfahrenswahlentscheidung unsicher. Sie wird im Folgenden als Ausprägung einer Zufallsvariablen $\tilde{x}$ modelliert, die sich additiv aus dem Erwartungswert $E[x]=\hat{x}$ und einem normalverteilten Störterm $\tilde{e}$ mit Erwartungswert Null und Varianz $\sigma^{2}$ zusammensetzt. ${ }^{7}$ Damit beträgt die nach der Verfahrenswahlentscheidung tatsächlich realisierte Leistungsmenge in (1)

$$
x=\widehat{x}+e
$$

und die erwarteten Kosten für Verfahren $i$ betragen:

$$
E\left[K_{i}\right]=F_{i}+c_{i} \cdot \widehat{x}
$$

Um den Einfluss der Risikoaversion des Entscheiders auf die Lösung des Verfahrenswahlproblems untersuchen zu können, nehmen wir an, dass sich die Präferenzen des Entscheiders durch die exponentielle Nutzenfunktion $U\left(K_{i}(x)\right)=-e^{r \cdot\left[-K_{t}(x)\right]}$ beschreiben lassen. Darin beschreibt der Parameter $r$ das Maß der konstanten absoluten Risikoaversion des Entscheiders. In Verbindung mit der Normalverteilungsannahme über den Störterm ermöglicht es die gewählte Form der Nutzenfunktion, den erwarteten Nutzen des Entscheiders aus einem gegebenen Verfahren $i$ durch das Sicherheitsäquivalent zu charakterisieren: ${ }^{8}$

$$
C E_{i}=-c_{i} \cdot \widehat{x}-F_{i}-\frac{1}{2} \cdot r \cdot c_{i}^{2} \cdot \sigma^{2}
$$

Demnach lässt sich der (Dis-)Nutzen des Entscheiders aus der Wahl des Verfahrens $i$ als Summe aus den erwarteten Kosten des Verfahrens und einer Risikoprämie darstellen, die durch den letzten Term in (4) gegeben ist. Die Risikoprämie stellt den über die erwarteten Kosten hinausgehenden Disnutzen des Entscheiders aus der Übernahme des Kostenrisikos dar. Sie ist um so grösser, je höher die Risikoaversion des Entscheiders und die Varianz der Kosten ausfallen. Dabei steigt die Varianz der Kosten von Verfahren $i, \operatorname{VAR}\left[K_{i}\right]=c_{i}^{2} \cdot \sigma^{2}$, linear in der Varianz des Störterms und quadratisch in der Höhe der variablen Stückkosten des gewählten Verfahrens.

Für die Lösung des Verfahrenswahlproblems bedarf es in diesem Rahmen nicht mehr nur eines einfachen Kostenvergleichs, sondern eines Vergleichs der aus den verschiedenen Verfahren resultierenden Sicherheitsäquivalente. Demnach gilt für zwei beliebig benachbarte Verfahren aus der geordneten Reihe aller Verfahren, dass Verfahren $i+1 \mathrm{dem}$ Verfahren $i$ dann vorzuziehen ist, wenn

$$
C E_{i+1}>C E_{i}
$$

also das aus der Wahl von Verfahren $i+1$ resultierende Sicherheitsäquivalent grösser ist als dasjenige, das bei Wahl von Verfahren $i$ erzielbar wäre. Vor diesem Hintergrund wollen wir im Folgenden untersuchen, wie sich die Mengenunsicherheit und die Risikoneigung des Entscheiders auf das zu lösende Verfahrenswahlproblem auswirken. 


\section{Verfahrenswahl bei Risiko}

\section{Optimales Verfahren bei Risikoneutralität}

In diesem Abschnitt betrachten wir zunächst die Verfahrenswahl eines risikoneutralen Entscheiders $(r=0)$. Entsprechend entfällt die Risikoprämie des Entscheiders in (4), so dass sich die Entscheidungsregel in (5) auf einen Vergleich der erwarteten Kosten der Verfahren reduziert. Danach ist Verfahren $i+1$ dem Verfahren $i$ vorzuziehen, wenn

$$
E\left[K_{i+1}\right]<E\left[K_{i}\right]
$$

also die erwarteten Kosten von Verfahren $i+1$ kleiner sind als die von Verfahren $i$. Setzt man für die erwarteten Kosten aus (3) ein, erhält man als Vorteilhaftigkeitsbedingung für die Wahl von Verfahren $i+1$ die Lösung

$$
\widehat{x}>\frac{F_{i+1}-F_{i}}{c_{i}-c_{i+1}} .
$$

Die Wahl des mit höheren Fixkosten verbundenen Verfahrens $i+1$ ist also genau dann vorteilhaft, wenn die erwartete Menge grösser ist als der Quotient aus den zusätzlichen Fixkosten und den ersparten variablen Kosten pro Stück. Dieselbe Break-Even-Menge ergibt sich als Standardlösung des Verfahrenswahlproblems bei Sicherheit, mit dem Unterschied, dass sich die Bedingung dort auf die tatsächliche und nicht auf die erwartete Menge bezieht. Für einen risikoneutralen Entscheider hat die Einführung von Unsicherheit also keinen Einfluss auf die Entscheidungsregel zur Lösung des Verfahrenswahlproblems. Es genügt, die für die tatsächliche Leistungsmenge geltende Entscheidungsregel auf die erwartete Leistungsmenge anzuwenden, um die optimale Lösung des Verfahrenswahlproblems zu implementieren.

Im Rahmen eines vollständigen Vergleichs aller verfügbaren Verfahren kann es neben den ausgeschlossenen Triviallösungen, in denen ein Verfahren von einem anderen direkt dominiert wird, auch Lösungen geben, in denen ein Verfahren durch die Kombination zweier benachbarter Verfahren dominiert wird. Diese Situation ist in Abb. 1 dargestellt, in der das Verfahren 2 durch die Kombination der Verfahren 1 und 3 dominiert wird.

Formal erkennt man diese Situation, indem man beginnend mit dem Verfahren, das die geringsten Fixkosten aufweist, der Reihe nach alle Schnittpunkte zwischen zwei benachbarten Verfahren berechnet. Ergibt sich dabei für ein Verfahren eine kleinere Break-EvenMenge als im vorangegangenen Berechnungsschritt, ist das zwischen den beiden betreffenden Schnittpunkten liegende Verfahren ineffizient. ${ }^{9}$ Bezeichne $\hat{x}_{i j}$ den Schnittpunkt zwischen Verfahren $i$ und $j$, so ist Verfahren $i$ genau dann ineffizient, wenn

$$
\widehat{x}_{i-1, i}>\widehat{x}_{i, i+1}
$$

Ist diese Bedingung erfüllt, muss unter Vernachlässigung des ineffizienten Verfahrens $i$ ein neuer Schnittpunkt zwischen den Verfahren $i-1$ und $i+1$ bestimmt werden, um das Verfahrenswahlproblem zu lösen. Entsprechend erkennt man auch in Abb. 1, dass $\hat{x}_{12}$ 


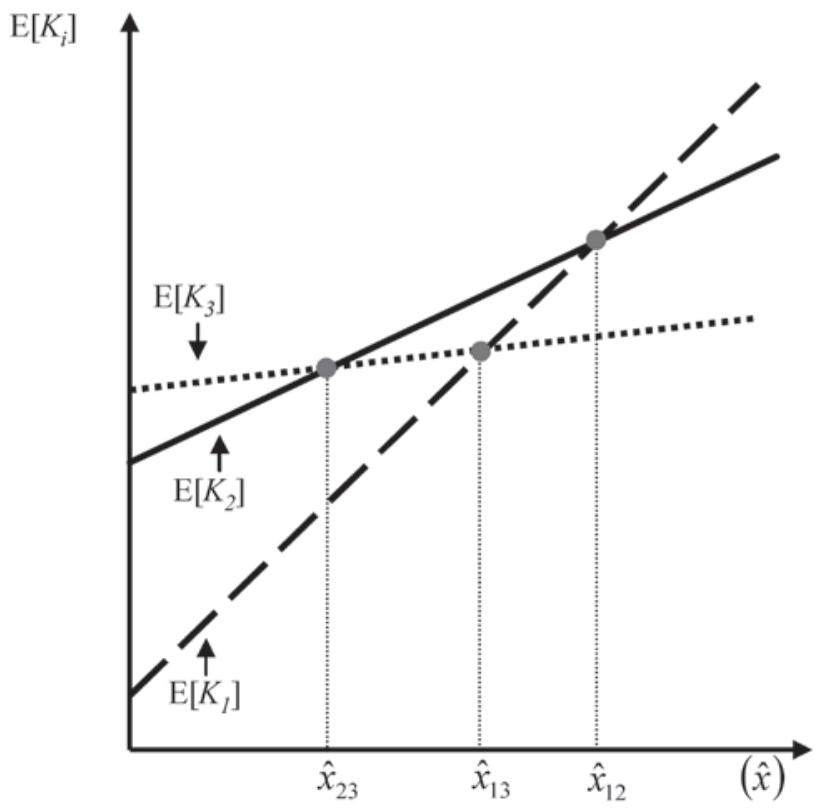

Abb. 1. Kombinierte Dominanz von Verfahren

grösser ist als $\hat{x}_{23}$, so dass einzig der Übergang zwischen den Verfahren 1 und 3 im Punkt $\hat{x}_{13}$ für die Bestimmung des effizienten Pfades relevant ist.

\section{Optimales Verfahren bei Risikoaversion}

Ist der Entscheider risikoavers $(r>0)$, kann das Verfahrenswahlproblem nicht mehr durch einen einfachen Vergleich der erwarteten Kosten gelöst werden, sondern es muss auch der Einfluss der Verfahrenswahl auf die Risikoprämie des Entscheiders berücksichtigt werden. Diese steigt gemäß (4) unabhängig vom gewählten Verfahren mit steigender Risikoaversion des Agenten $(r)$ und mit steigender Varianz der Leistungsmenge $\left(\sigma^{2}\right)$. Gleichzeitig steigt die Risikoprämie jedoch auch mit den variablen Kosten des Verfahrens. Da die variablen Stückkosten über die Kostenvarianz quadratisch in die Risikoprämie eingehen, steigt auch die marginale Risikoprämie in den variablen Stückkosten des Verfahrens, so dass die Risikoprämie mit den variablen Verfahrensstückkosten überproportional zunimmt.

Für die Verfahrenswahlentscheidung eines risikoaversen Entscheiders ergibt sich durch Einsetzen von (4) in (5) zunächst, dass dieser Verfahren $i+1$ gegenüber Verfahren $i$ bevorzugt, wenn die erwartete Leistungsmenge folgende Bedingung erfüllt:

$$
\widehat{x}>\frac{F_{i+1}-F_{i}}{c_{i}-c_{i+1}}-\frac{1}{2} \cdot r \cdot \sigma^{2} \cdot\left(c_{i+1}+c_{i}\right) .
$$

Der erste Teil der Bedingung in (8) entspricht der Bedingung bei Risikoneutralität in (6), so dass sich der Einfluss der Risikoaversion auf das Verfahrenswahlproblem ausschliesslich durch den zweiten Term in (8) bestimmt. Da dieser Term strikt positiv ist, verschiebt 
sich der Schnittpunkt zwischen zwei beliebigen Verfahren $i$ und $i+1$ unter Risikoaversion nach links. Ein risikoaverser Entscheider wählt also bereits bei einer geringeren erwarteten Leistungsmenge das Verfahren mit den höheren Fixkosten und den geringeren variablen Stückkosten als dies für einen risikoneutralen Entscheider optimal wäre.

Intuitiv erscheint dieses Ergebnis zunächst ein wenig überraschend, da man vermuten könnte, dass ein risikoaverser Entscheider das mit höheren Fixkosten verbundene Investitionsrisiko scheut und deshalb erst bei einer höheren Break-Even-Menge auf ein Verfahren mit geringeren variablen Stückkosten übergehen würde als bei Risikoneutralität. Diese Vermutung erweist sich jedoch bei einer rein kostenbasierten Betrachtungsweise als falsch.

Da die Risikoprämie unabhängig von der erwarteten Leistungsmenge ist, wirkt ihre Berücksichtigung bei gegebenen Verfahrenskosten zunächst wie eine Erhöhung der fixen Kosten des betrachteten Verfahrens. Die Höhe der Risikoprämie bestimmt sich bei gegebener Varianz und Risikoaversion ausschliesslich durch die Höhe der variablen Stückkosten des Verfahrens. Dabei werden Verfahren mit geringeren Fixkosten und entsprechend höheren variablen Stückkosten stärker belastet als solche mit höheren Fixkosten und kleineren variablen Stückkosten, so dass bei einem Übergang von Verfahren $i$ auf Verfahren $i+1$ nicht nur die variablen Kosten sinken, sondern auch die Risikoprämie variiert, und zwar um den Betrag

$$
\Delta R_{i+1}=\frac{1}{2} \cdot r \cdot \sigma^{2} \cdot\left(c_{i+1}^{2}-c_{i}^{2}\right)<0 .
$$

Die Risikoprämie sinkt also mit dem Übergang von Verfahren $i$ auf Verfahren $i+1$. Dieser Rückgang der Risikoprämie wirkt dem gleichzeitigen Fixkostenanstieg entgegen und senkt damit gewissermaßen die Einstiegskosten in die wirtschaftlichere Produktionsalternative.

Die Tatsache, dass der Entscheider das Risiko mengenbedingter Kostenschwankungen in seinem Entscheidungskalkül berücksichtigt, führt also im Ergebnis zunächst dazu, dass Verfahren mit geringeren variablen Kosten aufgrund ihrer geringeren Kostenvarianz relativ günstiger beurteilt werden als bei Risikoneutralität.

Sind die variablen Stückkosten des Verfahrens $i$ vergleichweise hoch, kann es sogar vorkommen, dass die Risikoprämie beim Übergang von Verfahren $i$ auf Verfahren $i+1$ stärker abnimmt als die fixen Verfahrenskosten zunehmen. Ist aber

$$
-\Delta R_{i+1}>F_{i+1}-F_{i}
$$

wird der in (8) definierte Schnittpunkt negativ, was nichts anders bedeutet, als dass Verfahren $i$ für alle erwarteten Mengen von Verfahren $i+1$ dominiert wird. Risikoaversion kann also im Extremfall dazu führen, dass Verfahren mit hohen variablen Kosten, die unter Risikoneutralität für kleine Leistungsmengen effizient wären, unter Risikoaversion von anderen Verfahren dominiert werden.

Umgekehrt stellt sich die Frage, ob auch Verfahren, die bei Risikoneutralität dominiert werden, unter Risikoaversion Element der effizienten Alternativenmenge sein können. Interessant ist dabei vor allem der Fall, in dem bei Risikoneutralität zwei Verfahren gemeinsam ein anderes Verfahren dominieren. Gemäß (7) und (6) wird Verfahren $i$ bei Risikoneutralität genau dann von den Verfahren $i-1$ und $i+1$ gemeinsam dominiert, wenn 
(9) $\Delta \widehat{x}_{i}=\frac{F_{i}-F_{i-1}}{c_{i-1}-c_{i}}-\frac{F_{i+1}-F_{i}}{c_{i}-c_{i+1}}>0$,

also die Schnittpunkte zwischen den benachbarten Verfahren keine aufsteigende Ordnung aufweisen. Damit kein Verfahren dominiert wird, muss auch bei Risikoaversion die geordnete Reihe der Schnittpunkte aller benachbarten Verfahren eine aufsteigende Ordnung aufweisen. Dabei sind die Schnittpunkte allerdings gemäß (8) und nicht gemäß (6) zu bestimmen. Demnach ist Verfahren $i$ effizient, wenn folgende Bedingung erfüllt ist

$$
\frac{1}{2} \cdot r \cdot \sigma^{2} \cdot\left(c_{i-1}-c_{i+1}\right)>\frac{F_{i}-F_{i-1}}{c_{i-1}-c_{i}}-\frac{F_{i+1}-F_{i}}{c_{i}-c_{i+1}}=\Delta \widehat{x}_{i}
$$

Da die linke Seite von (10) strikt positiv ist, ist Verfahren $i$ bei Risikoaversion immer dann effizient, wenn $\Delta \hat{x}_{i} \leq 0$. Das ist gemäß (9) genau dann der Fall, wenn Verfahren $i$ auch bei Risikoneutralität effizient ist. Diese Beziehung lässt sich jedoch nicht umkehren. Die Ungleichung in (10) kann nämlich auch dann erfüllt sein, wenn $\Delta \hat{x}_{i}>0$; also Verfahren $i$ bei Risikoneutralität durch die Kombination seiner benachbarten Verfahren dominiert wird.

Es können also Entscheidungssituationen auftreten, in denen ein risikoaverser Entscheider ein Verfahren wählt, dass bei Risikoneutralität nicht zur Menge der effizienten Alternativen zählt. Diese Situation ist gemäß Bedingung (10) bei gegebener Alternativenmenge umso wahrscheinlicher, je stärker die Risikoaversion des Entscheiders und je grösser die Varianz der Leistungsmenge ausfallen, sie ist in Abbildung 2 anhand eines Beispiels illustriert.

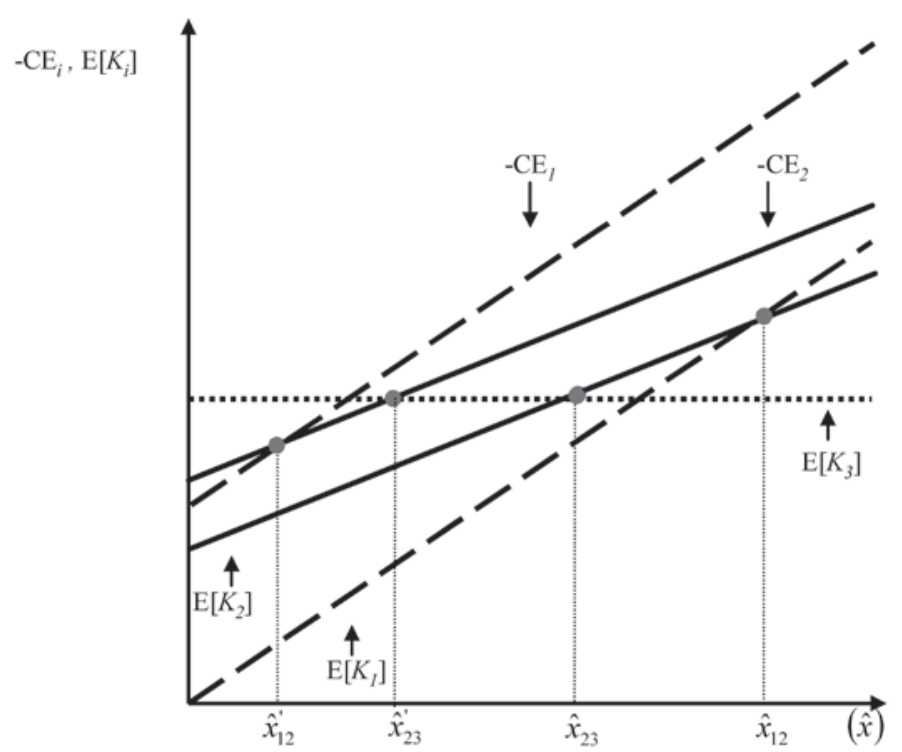

Abb. 2. Dominanzumkehr unter Risikoaversion 
Das Beispiel geht von drei Verfahren aus, wobei vereinfachend unterstellt wird, dass Verfahren 1 nur variable Kosten und Verfahren 3 nur Fixkosten verursacht. Bei Risikoneutralität ergeben sich die Schnittpunkte $\hat{x}_{12}$ und $\hat{x}_{23}$ zwischen den erwarteten Kosten der benachbarten Verfahren. Dabei ist wie in Abb. $1 \hat{x}_{12}>\hat{x}_{23}$, so dass Verfahren 2 von der Kombination seiner benachbarten Verfahren 1 und 3 dominiert wird. Während Verfahren 3 wegen des fehlenden Kostenrisikos bei Risikoaversion gleich beurteilt wird, müssen für die Ermittlung der Sicherheitsäquivalente von Verfahren 1 und 2 zu den erwarteten Kosten die entsprechenden Risikoprämien hinzuaddiert werden. Dabei ist die Risikoprämie von Verfahren 1 aufgrund der höheren Kostenvarianz grösser als die von Verfahren 2. Entsprechend verschiebt sich in Abb. 1 die Funktion $-C E_{1}$ gegenüber $\mathrm{E}\left[K_{1}\right]$ stärker nach oben als $-C E_{2}$ gegenüber $\mathrm{E}\left[K_{2}\right]$. Die neuen Schnittpunkte zwischen den benachbarten Verfahren ergeben sich schließlich als $\hat{x}_{12}^{\prime}$ und $\hat{x}_{23}^{\prime}$, wobei man erkennt, dass $\hat{x}_{23}^{\prime}>\hat{x}_{12}^{\prime}$, so dass der risikoaverse Entscheider für erwartete Mengen zwischen den beiden Schnittpunkten das Verfahren 2 wählen würde.

\section{Erweiterungen des Grundmodells}

\section{Zur Separierbarkeit von Kosten- und Nutzenbetrachtungen}

Die bisherige Analyse beschränkt sich auf eine rein kostenbasierte Betrachtungsweise, während der mit dem Einsatz des Verfahrens erzielbare Nutzen unberücksichtigt blieb. Auch wenn diese Vorgehensweise dem Standard einschlägiger Lehrbücher entspricht ${ }^{10}$, ist sie nur dann zulässig, wenn die Berücksichtigung des Nutzens zu den gleichen Ergebnissen wie die kostenbasierte Betrachtungsweise führt, also das eigentliche Verfahrenswahlproblem von der Nutzungsentscheidung separiert werden kann.

$\mathrm{Ob}$ diese Bedingung bei Risiko stets erfüllt ist, soll im Folgenden untersucht werden. Dazu betrachten wir in Erweiterung unseres Modells nun eine Situation, in der die Leistungsmenge des Verfahrens in (1) der Absatzmenge eines Endproduktes entspricht, das am Markt zu einem konstanten Preis $p$ verkauft werden kann. Dabei gelte vereinfachend $p>c_{1}$ und $F_{1}=0$; so dass eine Produktion mit dem unwirtschaftlichsten aller Verfahren bereits für kleine Absatzmengen grundsätzlich lohnend erscheint ${ }^{11}$ und somit auch der Stückdeckungsbeitrag, $d_{i}=p-c_{i}$, für alle Verfahren positiv ist. Alle übrigen Modellannahmen entsprechen denen, die in Abschnitt B getroffen wurden.

Die relevante Größe für die Verfahrenswahl ist unter diesen Bedingungen das Sicherheitsäquivalent des erwarteten Nutzens aus dem Erfolgsbeitrag von Verfahren $i, D_{i}(x)=$ $d_{i} \cdot x-F_{i}$. Für Verfahren $i$ erhält man das Sicherheitsäquivalent

$$
C E_{i}^{+}=d_{i} \cdot \widehat{x}-F_{i}-\frac{1}{2} \cdot r \cdot d_{i}^{2} \cdot \sigma^{2}
$$

Die Struktur des Ausdrucks in (11) entspricht derjenigen in (4) mit dem Unterschied, dass an die Stelle der variablen Kosten die Deckungsbeitragsfunktion und an die Stelle der Kostenvarianz die Varianz des Erfolgsbeitrages tritt. Diese beträgt $\operatorname{VAR}\left[D_{i}\right]=d_{i}^{2} \cdot \sigma^{2}$ und steigt quadratisch im Stückdeckungsbeitrag $d_{i}$. Analog zu der Entscheidungsregel in (5) 
ist Verfahren $i+1$ dem Verfahren $i$ vorzuziehen, wenn $C E_{i+1}^{+}>C E_{i}^{+}$. Im Falle eines risikoneutralen Entscheiders $(r=0)$ ergibt sich daraus als Vorteilhaftigkeitsschwelle eine erwartete Menge von

$$
\widehat{x}>\frac{F_{i+1}-F_{i}}{d_{i+1}-d_{i}}=\frac{F_{i+1}-F_{i}}{c_{i}-c_{i+1}} .
$$

Da der Absatzpreis unabhängig von der Absatzmenge ist, entspricht die Differenz der Stückdeckungsbeiträge der Differenz der Stückkosten, so dass sich dieselbe Lösung wie bei einer kostenbasierten Betrachtungsweise ergibt. Bei Risikoneutralität führt also ein kostenbasiertes Entscheidungskalkül zum gleichen Ergebnis wie eine nutzenbasierte Entscheidungsregel, so dass sich ein risikoneutraler Entscheider ohne Beschränkung der Allgemeinheit auf eine reine Kostenbetrachtung beschränken kann.

Demgegenüber bevorzugt ein risikoaverser Entscheider Verfahren $i+1$ gegenüber Verfahren $i$, wenn die erwartete Absatzmenge folgenden Schwellenwert übertrifft:

$$
\widehat{x}>\frac{F_{i+1}-F_{i}}{c_{i}-c_{i+1}}+\frac{1}{2} \cdot r \cdot \sigma^{2} \cdot\left(d_{i}+d_{i+1}\right)
$$

Die Entscheidungsregel in (13) unterscheidet sich von der Entscheidungsregel bei Risikoneutralität. Im Gegensatz zu einer rein kostenbasierten Betrachtungsweise kommt es aber zu einer Rechtsverschiebung der Break-Even-Menge zwischen Verfahren $i$ und $i+1$. Ein risikoaverser Entscheider geht also bei gegebenen Verfahrenskosten erst bei einer höheren erwarteten Menge auf Verfahren $i+1$ über als ein risikoneutraler Entscheider.

Dieses Ergebnis steht im klaren Widerspruch zu dem Ergebnis einer rein kostenbasierten Betrachtungsweise, bei der die Risikoaversion des Entscheiders gemäß (8) eine Linksverschiebung der Break-Even-Menge zwischen Verfahren $i$ und $i+1$ bewirkt. Das folgende Zahlenbeispiel veranschaulicht die Ergebnisunterschiede: Gegeben seien zwei Verfahren mit den Kostenfunktionen $K_{1}(x)=20 x+1000$ und $K_{2}(x)=5 x+4000$. Die Varianz des Störterms betrage $\sigma^{2}=100$, der Risikoaversionskoeffizient $r=0.1$ und der Preis $p=25$. Als Benchmarklösung würde sich bei Risikoneutralität eine Absatzmengenschwelle von $\hat{x}=200$ für die Vorteilhaftigkeit von Verfahren 2 ergeben. Bei Risikoaversion und kostenorientierter Betrachtungsweise sinkt die kritische Absatzmenge auf $\hat{x}=75$, während sich bei einer Berücksichtigung der Deckungsbeiträge der Verfahren ein Schnittpunkt von $\hat{x}=325$ ergeben würde.

Die abweichenden Resultate bei deckungsbeitrags- und kostenorientierter Betrachtungsweise lassen sich mit der unterschiedlichen Bewertung des Risikos von Absatzmengenschwankungen erklären. In beiden Fällen wirkt die Berücksichtigung des Risikos bei gegebenem Verfahren zunächst wie eine Erhöhung der Fixkosten des Verfahrens $i$. Während aber das Kostenrisiko beim Übergang von Verfahren $i$ auf $i+1$ abnimmt und somit tendenziell zu einer Begünstigung von Verfahren mit hohen Fixkosten und geringen variablen Stückkosten führt, steigen die Deckungsbeiträge und damit auch das Deckungsbeitragsrisiko wegen der sukzessiven Abnahme der variablen Stückkosten an, und zwar um

$$
\Delta R_{i+1}^{+}=\frac{1}{2} \cdot r \cdot \sigma^{2} \cdot\left(d_{i+1}^{2}-d_{i}^{2}\right)>0
$$


Diese Zunahme der Risikoprämie verstärkt den mit dem Übergang von Verfahren $i$ auf Verfahren $i+1$ verbundenen Fixkostenanstieg und macht damit Verfahren mit hohen Fixkosten tendenziell unattraktiver, so dass es für die Bereitschaft zum Einstieg in eine verbesserte Technologie einer höheren erwarteten Absatzmenge bedarf.

Dieses Ergebnis deckt sich mit der im vorigen Abschnitt formulierten Vermutung, dass ein risikoaverser Entscheider hohe Investitionen eher scheut und deshalb erst bei einer höheren Break-Even-Menge auf ein Verfahren mit geringeren variablen Stückkosten übergeht als ein risikoneutraler Entscheider. Die Vermutung erweist sich in der vorliegenden Situation als zutreffend, da der Entscheider sich bei Verwendung aller verfügbaren Informationen vernünftigerweise an seinem Einkommensrisiko orientiert und nicht nur das Kostenrisiko betrachtet. So wird im Falle einer deckungsbeitragsorientierten Betrachtungsweise zutreffend berücksichtigt, dass ein Verfahren mit geringeren variablen Kosten zwar den Stückdeckungsbeitrag und damit die Einkommenschancen erhöht, gleichzeitig aber über die höheren Fixkosten hinaus auch das Einkommensrisiko erhöht, da sich Absatzmengenschwankungen naturgemäß stärker auf das Ergebnis auswirken, und dies obwohl das Kostenrisiko bei isolierter Betrachtungsweise gleichzeitig sinkt.

Ist der Nutzen des Verfahrenseinsatzes bekannt und variiert dieser mit der Einsatzmenge, führt bei Risikoaversion nur eine deckungsbeitragsorientierte Betrachtungsweise zu einer korrekten Verfahrenswahlentscheidung. Das bedeutet aber nicht, dass eine Verfahrenswahlentscheidung auf Basis einer isolierten Betrachtung des Kostenrisikos in keinem Fall zum richtigen Ergebnis führen kann. Ist der Nutzen nämlich unabhängig von der Leistungsmenge und damit sicher, führt die kostenorientierte Betrachtungsweise stets zum korrekten Ergebnis. Das ist zum Beispiel dann der Fall, wenn es um die kostengünstigste Bereitstellung der Ressourcen für einen Auftrag mit fixem Gesamtentgelt geht. Schwieriger zu beurteilen sind Situationen, in denen die Kosten bekannt aber der Nutzen und sein Zusammenhang mit der Leistungsmenge schwer abzuschätzen sind, wie dies etwa bei der Auswahl zwischen unterschiedlichen Telefontarifen und Versicherungspolicen mit unterschiedlich hohen Prämien und Selbstbehalten der Fall ist.

\section{Verfahrenswahl und Delegation}

Häufig werden Entscheidungen über Maßnahmen, die das operative Geschäft betreffen, von der Unternehmensleitung auf die Bereichsebene delegiert, während die Unternehmensleitung sich auf Entscheidungen über Maßnahmen mit längerfristigem Charakter beschränkt. In Bezug auf unser Verfahrenswahlproblem würde diese Konstellation bedeuten, dass die Unternehmensleitung das Verfahren bestimmt und das Bereichsmanagement für die Absatzmenge verantwortlich ist. In diesem Zusammenhang stellt sich die Frage, ob und in welcher Form die Delegation der Absatzpolitik an das Bereichsmanagement Rückwirkungen auf die Verfahrenswahlentscheidung der Zentrale hat. Im Folgenden erweitern wir daher unser bisheriges Modell zu einem einfachen linearen PrinzipalAgenten-Ansatz. ${ }^{12}$

Ein risikoneutraler Unternehmenseigner (der Prinzipal) sieht sich dem in Abschnitt B beschriebenen Verfahrenswahlproblem gegenüber. Im Unterschied zum Grundmodell ist das operative Geschäft jedoch an einen risiko- und arbeitsaversen Manager (der Agent) delegiert, der durch seinen persönlichen Arbeitseinsatz $a \in \mathfrak{R}_{+}$die Absatzmenge erhöhen 
kann. Entsprechend setzt sich die unsichere Nachfragemenge nunmehr aus drei Komponenten zusammen, dem erwarteten Basisabsatz $\hat{x}$, dem Beitrag des Agenten und der Realisation des normalverteilten Störterms:

$$
x=\widehat{x}+a+e .
$$

Dabei sei angenommen, dass der Prinzipal die tatsächliche Nachfrage beobachten kann und ihm auch deren Struktur, der Basisabsatz $\hat{x}$ und die Verteilung des Störterms bekannt sind. Der Arbeitseinsatz des Agenten ist hingegen für den Prinzipal weder direkt beobachtbar noch kann er angesichts des Störterms aus der beobachteten Nachfrage Rückschlüsse auf den Arbeitseinsatz des Agenten ziehen.

Die Präferenzen des Agenten lassen sich durch eine exponentielle Nutzenfunktion ana$\log$ zu der des Entscheiders in Abschnitt B beschreiben. Der Arbeitseinsatz verursacht dem Agenten Arbeitsleid, dessen Geldäquivalent durch die quadratische Funktion

$$
C(a)=\frac{1}{2} a^{2}
$$

gegeben sei. Die persönlichen Kosten des Agenten begründen einen Interessenkonflikt zwischen Prinzipal und Agent. Der Prinzipal ist an einem möglichst hohen Arbeitseinsatz interessiert, den der Agent möglichst vermeiden möchte. Da die Unbeobachtbarkeit des Arbeitseinsatzes eine vertragliche Vereinbarung über den Einsatz des Agenten ausschließt, muss der Prinzipal dem Agenten einen erfolgsabhängigen Entlohnungsvertrag anbieten, um den Agenten zum Arbeitseinsatz zu motivieren. Dieser Vertrag habe im Falle der Wahl von Verfahren $i$ folgende Gestalt:

$$
s_{i}(x)=w+v \cdot d_{i} \cdot x .
$$

Darin bezeichnet der Parameter $w$ ein erfolgsunabhängiges Fixum und der zweite Term den erfolgsabhängigen Teil der Entlohnung. Diese setzt sich multiplikativ zusammen aus dem Bonuskoeffizienten $v$ und dem Gesamtdeckungsbeitrag $d_{i} \cdot x$ als Bemessungsgrundlage der Entlohnung. ${ }^{13}$ Aufgrund der exponentiellen Nutzenfunktion lässt sich auch hier der Erwartungsnutzen des Agenten bei Wahl von Verfahren $i$ mittels seines Sicherheitsäquivalentes abbilden:

$$
C E A_{i}=w+v \cdot d_{i} \cdot(\widehat{x}+a)-\frac{1}{2} a^{2}-\frac{1}{2} \cdot r \cdot v^{2} \cdot d_{i}^{2} \cdot \sigma^{2}
$$

Es setzt sich zusammen aus der erwarteten Entlohnung, den Kosten des Arbeitseinsatzes und der Risikoprämie, die den Disnutzen des Agenten aus der risikobehafteten Entlohnung erfasst. Diese ergibt sich als Produkt aus dem Risikoaversionskoeffizienten $(r)$ und der Varianz der Entlohnung, die sich ihrerseits aus dem Bonuskoeffizienten $(v)$, dem Deckungsbeitrag des gewählten Verfahrens $\left(d_{i}\right)$ und der Varianz der Absatzmenge $\left(\sigma^{2}\right)$ zusammensetzt.

Das Problem des Prinzipals besteht nun darin, zunächst das Verfahren zu wählen und anschließend einen Entlohnungsvertrag zu entwerfen. Die Lösung des Problems beginnt auf der zweiten Stufe mit der Gestaltung des Anreizvertrages. Hier maximiert der Prinzi- 
pal die erwartete Differenz zwischen dem Erfolgsbeitrag des Verfahrens $i$ und der Entlohnung des Agenten,

$$
\max _{w, v} U P_{i}=E\left[D_{i}(x)\right]-E\left[s_{i}(x)\right]
$$

unter den Nebenbedingungen

$$
a=\arg \max _{a^{\prime}} C E A_{i}\left(a^{\prime}\right)
$$

$$
C E A \geq \underline{U}
$$

Die erste Bedingung in (16) ist die Anreizbedingung. Sie besagt, dass der Agent seinen Arbeitseinsatz bei gegebenen Entlohnungsparametern durch eine Maximierung seines Sicherheitsäquivalentes bestimmt. Die zweite Restriktion in (17) ist die Teilnahmebedingung des Agenten. Sie stellt sicher, dass das Sicherheitsäquivalent des Agenten mindestens seinem aus alternativen Beschäftigungsmöglichkeiten erzielbaren Reservationsnutzen $\underline{U}$ entspricht und er infolgedessen den Vertrag akzeptiert.

Da der Prinzipal den Vertrag entwirft, wird er diesen so gestalten, dass im Optimum beide Bedingungen als Gleichung erfüllt sind. Für die Anreizbedingung erhält man durch Maximierung von $C E A_{i}$ bezüglich $a$ zunächst den optimalen Arbeitseinsatz des Agenten bei gegebener Bonusrate und Verfahren $i$. Er beträgt $a_{i}^{*}=v \cdot d_{i}$ und steigt demnach in der Bonusrate $(v)$ und im Deckungsbeitrag des Verfahrens $\left(d_{i}\right)$ : Setzt man für die erwartete Entlohnung des Agenten aus der Teilnahmebedingung ein und berücksichtigt die Anreizbedingung, vereinfacht sich das Problem des Prinzipals zu:

$$
\max _{v} U P_{i}=d_{i} \cdot \widehat{x}-F_{i}+d_{i}^{2} \cdot\left(v-\frac{1}{2} \cdot\left(1+r \cdot \sigma^{2}\right) \cdot v^{2}\right)-\underline{U} .
$$

Gemäß (18) maximiert der Prinzipal den erwarteten Erfolgsbeitrag des Verfahrens zuzüglich des Nettobeitrags des Agenten zum Unternehmenserfolg. Dieser ist durch die letzten beiden Terme in (18) gegeben, wobei der vorletzte Term die Differenz zwischen den durch den Einsatz des Agenten erwirtschafteten Deckungsbeiträgen, den persönlichen Kosten und der Risikoprämie angibt. ${ }^{14}$ Als optimale Bonusrate erhält man schließlich:

$$
v^{*}=\frac{1}{1+r \sigma^{2}}
$$

Diese Lösung entspricht dem Standardfall des LEN-Modells ${ }^{15}$ und besagt, dass die optimale Bonusrate um so kleiner ausfällt, je risikoaverser der Agent ist und je grösser die Varianz der Absatzmenge ausfällt.

Interessanterweise ist angesichts der zugrunde gelegten Entlohnungsfunktion zwar der gleichgewichtige Arbeitseinsatz, nicht aber die optimale Bonusrate abhängig von der Wahl des Verfahrens durch den Prinzipal. Würde man den Bonus des Agenten statt an den Deckungsbeitrag des Verfahrens lediglich an die Absatzmenge knüpfen, erhielte man eine verfahrensabhängige Bonusrate in Höhe von $v^{* *}=d_{i} \cdot v^{*}$. Da in diesem Fall jedoch der 
Arbeitseinsatz des Agenten $a^{* *}=v^{* *}$ betrüge, ergibt sich in beiden Fällen derselbe gleichgewichtige Arbeitseinsatz.

Unter Berücksichtigung der optimalen Bonusrate in (19) erzielt der Prinzipal bei der Wahl von Verfahren $i$ einen erwarteten Nettoerfolg in Höhe von

$$
U P_{i}^{*}=d_{i} \cdot \widehat{x}-F_{i}+\frac{1}{2} \cdot d_{i}^{2} \cdot v^{*}-\underline{U} .
$$

Der Einsatz des Agenten beschert also dem Prinzipal einen über den Erfolgsbeitrag des Verfahrens $i$ hinausgehenden Nettobeitrag in Höhe von $\frac{1}{2} \cdot d_{i}^{2} \cdot v^{*}-\underline{U}>0 .{ }^{16}$ Der Nettobeitrag des Agenten steigt also mit dem Deckungsbeitrag des Verfahrens, so dass Verfahren mit geringeren variablen Stückkosten und höheren Fixkosten tendenziell günstiger beurteilt werden als solche mit höheren variablen Stückkosten aber geringeren Fixkosten. Ohne den Beitrag des Agenten würde der erwartete Überschuss des Prinzipals in (20) dem erwarteten Erfolgsbeitrag des Verfahrens entsprechen, so dass der Prinzipal seine Verfahrenswahl wie der risikoneutrale Entscheider im Grundmodell treffen würde. Angesichts des Delegationsproblems bevorzugt der Prinzipal jedoch Verfahren $i+1$ gegenüber Verfahren $i$ bereits dann, wenn der erwartete Basisabsatz folgende Schwelle überschreitet:

$$
\widehat{x}>\frac{F_{i+1}-F_{i}}{c_{i}-c_{i+1}}-\left(d_{i}+d_{i+1}\right) \cdot \frac{1}{2} \cdot v^{*}
$$

Gemäß (21) kommt es also bei korrekter Berücksichtigung der Delegationsbeziehung zu einer Linksverschiebung des Break-Even-Punktes zwischen Verfahren $i$ und $i+1$, so dass Verfahren mit höheren Fixkosten und geringeren variablen Kosten bereits bei kleineren erwarteten Absatzmengen attraktiv werden als ohne den Beitrag des Agenten. Der Betrag der Verschiebung entspricht dem mittleren gleichgewichtigen Arbeitseinsatz des Agenten über beide Verfahren, $\left(a_{i}^{*}+a_{i+1}^{*}\right) / 2$. Darin kommt die Tatsache zum Ausdruck, dass der Agent durch seinen Arbeitseinsatz die Absatzmenge erhöht und dadurch bereits bei einem geringeren erwarteten Basisabsatz den Verfahrensübergang erlaubt. Dieser Effekt ist um so stärker, je höher die Deckungsbeiträge der betrachteten Verfahren und damit der gleichgewichtige Arbeitseinsatz des Agenten ausfällt.

Als Zwischenfazit lässt sich festhalten, dass die Frage der Verfahrenswahl im Rahmen von Delegationsbeziehungen selbst dann nicht unabhängig von der Lösung des nachgeordneten Anreizproblems betrachtet werden kann, wenn der Prinzipal risikoneutral ist. Andernfalls besteht die Gefahr, dass durch eine suboptimale Verfahrenswahl der maximal mögliche Unternehmensgewinn verfehlt wird.

\section{E. Zusammenfassung}

Dieser Beitrag untersucht das Verfahrenswahlproblem bei Risiko. Im Mittelpunkt steht dabei die Frage, wie sich Unsicherheit über die Leistungsmenge und die Risikoneigung des Entscheiders auf die Lösung des Verfahrenswahlproblems auswirken. Dabei stellen wir zunächst fest, dass die unter Sicherheit abgeleiteten Entscheidungsregeln für die Ver- 
fahrenswahl bei unsicherer Leistungsmenge und Risikoneutralität grundsätzlich ihre Gültigkeit behalten. Dies gilt jedoch nicht mehr, wenn ein risikoaverser Entscheider unterstellt wird. Wird der Verfahrensvergleich auf Basis der Kosten durchgeführt, bewirkt die Risikoaversion einen früheren Übergang auf Verfahren mit höheren Fixkosten und geringeren variablen Stückkosten als bei Sicherheit, weil die Risikoprämie in den variablen Kosten steigt und damit Verfahren mit geringen variablen Kosten attraktiver macht. Dabei kann es vorkommen, dass Verfahren, die unter Risikoneutralität effizient sind, bei Risikoaversion von anderen Verfahren dominiert werden. Es ist aber ebenso möglich, dass Verfahren die unter Risikoneutralität von einer Kombination anderer Verfahren dominiert wurden, bei Risikoaversion zur Menge der effizienten Alternativen zählen.

In einer Erweiterung unseres Grundmodells lösen wir das Verfahrenswahlproblem auf der Basis von Deckungsbeiträgen. Dabei stellen wir fest, dass bei Risikoaversion nunmehr Verfahren mit geringen variablen Stückkosten attraktiver werden als bei Risikoneutralität. Der Unterschied zwischen deckungsbeitrags- und kostenorientierter Betrachtungsweise lässt sich mit der unterschiedlichen Bewertung des Risikos von Absatzmengenschwankungen erklären. Das Deckungsbeitragsrisiko steigt nämlich mit der Abnahme der variablen Stückkosten an, so dass Verfahren mit geringeren variablen Stückkosten nicht nur höhere Fixkosten, sondern auch eine höhere Risikoprämie aufweisen. Die Erweiterung zeigt, dass eine Verfahrenswahlentscheidung auf Basis einer isolierten Betrachtung des Kostenrisikos nur dann zum richtigen Ergebnis führen kann, wenn der Nutzen unabhängig von der Leistungsmenge und damit sicher ist. Andernfalls führt nur eine deckungsbeitragsorientierte Betrachtung zum richtigen Ergebnis.

In einer zweiten Erweiterung unseres Grundmodells untersuchen wir das Verfahrenswahlproblem im Kontext eines einfachen Prinzipal-Agenten-Modells, bei dem der Prinzipal die Verfahrenswahlentscheidung trifft und der Agent mit seinem Arbeitseinsatz die Absatzmenge erhöhen kann. Die Analyse zeigt, dass das Verfahrenswahlproblem trotz Risikoneutralität des Prinzipals nicht unabhängig von der Lösung des Anreizproblems gelöst werden kann, da andernfalls die Break-Even-Menge für den Verfahrensübergang überschätzt wird. Der Beitrag des Agenten macht nämlich Verfahren mit höheren Deckungsbeiträgen tendenziell attraktiver, so dass der Übergang auf Verfahren mit geringeren variablen Stückkosten wiederum früher erfolgt als im Standardfall.

Unsere Resultate zeigen, dass die unter Sicherheit abgeleiteten Entscheidungsregeln nicht ohne weiteres auf Entscheidungssituationen unter Risiko übertragen werden können. Eine optimale Lösung des Verfahrenswahlproblems erfordert nicht nur eine Berücksichtigung der Risikoneigung des Entscheiders, sondern auch eine korrekte Berücksichtigung der Rahmenbedingungen des Entscheidungsproblems.

\section{Anhang: Verfahrenswahl bei allgemeiner Nutzenfunktion}

In diesem Anhang wird gezeigt, dass die für den Fall der konstanten absoluten Risikoaversion hergeleiteten Zusammenhänge auch für risikoaverse Entscheider mit beliebiger Nutzenfunktion gültig sind. Dazu bezeichnen wir im Folgenden die Nutzenfunktion des Entscheiders mit $U(y)$ und nehmen lediglich an, dass $U^{\prime}(y)>0, U^{\prime \prime}(y)<0$, die Nutzenfunktion des Entscheiders also strikt konkav in $y$ ist. 
Betrachten wir zunächst den Fall eines mengenunabhängigen Verfahrensnutzen. Bei einem gegebenem Anfangsvermögen $V_{0}$ und einem Verfahrensnutzen $D$ beträgt der erwartete Nutzen des Entscheiders bei Wahl von Verfahren $i$

$$
E\left[U_{i}\right]=E\left[U\left(V_{0}+D-K_{i}(x)\right)\right]
$$

Die Leistungsmenge $x$ setzt sich gemäß (2) additiv aus dem Erwartungswert $\hat{x}$ und einem Störterm ẽ mit Erwartungswert Null und Varianz $\sigma^{2}$ zusammen. Abweichend von Abschnitt B unterstellen wir jedoch keine bestimmte Verteilung, sondern nehmen lediglich an, dass der Störterm eine symmetrische Dichte $\phi(e)$ auf dem geschlossenen Intervall $[\underline{e}, \bar{e}]$ besitzt, wobei $\underline{e}=-\bar{e}$. Weiter gelte $\hat{x}+\underline{e} \geq 0$, um negative Mengen auszuschließen. Um die Analyse auf nicht triviale Verfahrenswahlprobleme zu beschränken, gelte überdies, dass die Menge aller möglichen Erwartungswerte einen kritischen Wert $\hat{x}=\hat{x}_{i, i+1}^{\prime}$ enthält, so dass

$$
E\left[U_{i}\right] \gtreqless E\left[U_{i+1}\right] \quad \text { wenn } \quad \widehat{x} \gtreqless \widehat{x}_{i, i+1}^{*} .
$$

Zunächst gilt es zu zeigen, dass der Schnittpunkt der Erwartungsnutzen aus zwei benachbarten Verfahren bei mengenunabhängigem Verfahrensnutzen von der Lösung bei Risikoneutralität nach unten abweicht. Dazu bestimmen wir die Erwartungsnutzen an der Stelle $\widehat{x}=\widehat{x}_{i, i+1}=\frac{F_{i+1}-F_{i}}{c_{i}-c_{i+1}}$ und erhalten wegen $E\left[K_{i+1}\left(\hat{x}_{i, i+1}\right)\right]=E\left[K_{i}\left(\hat{x}_{i, i+1}\right)\right]=\hat{K}$ für die Erwartungsnutzen der benachbarten Verfahren $i$ und $i+1$ die Ausdrücke:

$$
\begin{aligned}
& \left.E\left[U_{i}\right]\right|_{\widehat{x}=\widehat{x}_{i, i+1}}=E\left[U\left(V_{0}+D-\widehat{K}-c_{i} \cdot e\right)\right] \\
& \left.E\left[U_{i+1}\right]\right|_{\widehat{x}=\widehat{x}_{i, i+1}}=E\left[U\left(V_{0}+D-\widehat{K}-c_{i+1} \cdot e\right)\right]
\end{aligned}
$$

Die Ausdrücke in (23) und (24) besitzen die gleiche Struktur und unterscheiden sich lediglich durch den letzten Term im Argument der Nutzenfunktion. Etwas allgemeiner betrachtet geht es darum, den erwarteten Nutzen aus einer affin linearen Transformation $y(e)=a+b \cdot e$ einer Zufallsvariablen $e$ mit Erwartungswert Null zu bestimmen, wobei $a=V_{0}+D-\hat{K}$ und $b \in\left\{c_{i}, c_{i+1}\right\}$. Das Sicherheitsäquivalent des Erwartungsnutzens aus dieser Transformation ist allgemein wie folgt definiert

$$
C E(y(e))=a-\delta(b)=U^{-1} E[U(y(e))]
$$

Darin bezeichnet $\delta(b)$ die Risikoprämie der Einkommenslotterie $y(e)$ für die Nutzenfunktion $U(y)$. Demnach unterscheiden sich bei gegebenem Basiseinkommen $a$ zwei unterschiedliche Lotterien nur durch die Höhe ihrer Risikoprämien. Nun lässt sich unter Verwendung einer Taylor-Approximation zweiter Ordnung an der Stelle $b=0$ die Risikoprämie durch folgenden Ausdruck approximieren ${ }^{17}$ :

$$
\delta(b) \approx-\frac{U(y)^{\prime \prime}}{U(y)^{\prime}} \cdot \frac{\sigma^{2}}{2} \cdot b^{2} .
$$

Aufgrund der strikt konkaven Nutzenfunktion ist die Risikoprämie stets positiv. Da der Ausdruck in (25) quadratisch in dem Parameter $b$ steigt, ist die Risikoprämie um so höher, 
je stärker das Gewicht auf dem Störterm ausfällt. Diese Aussage gilt unabhängig vom Vorzeichen des Parameters $b$. Für den Vergleich der beiden Ausdrücke in (23) und (24) folgt damit unmittelbar, dass $\delta\left(c_{i}\right)>\delta\left(c_{i+1}\right)$ und damit $\left.E\left[U_{\mathrm{i}+1}\right]\right|_{\hat{x}=\hat{x}_{i, i+1}}>\left.E\left[U_{i}\right]\right|_{\hat{x}=\hat{x}_{i, i+1}}$.

Wie im Falle der konstanten absoluten Risikoaversion verschiebt sich damit der für den Verfahrensübergang relevante Schnittpunkt $\hat{x}_{i, i+1}^{*}$ gegenüber dem Schnittpunkt bei Risikoaversion $\hat{x}_{i, i+1}$ nach links, weil das Verfahren mit den höheren variablen Stückkosten das größere Kostenrisiko beinhaltet und Verfahren $i+1$ damit relativ attraktiver macht.

Betrachtet man schließlich den Fall eines mengenabhängigen Verfahrensnutzen, ergibt sich bei einem gegebenem Anfangsvermögen $V_{0}$ und einem konstanten Absatzpreis $p$ für den erwarteten Nutzen des Entscheiders bei Wahl von Verfahren $i$

$$
E\left[U_{i}\right]=E\left[U\left(V_{0}+p \cdot x-K_{i}(x)\right)\right]
$$

Wertet man auch diesen Ausdruck an der Stelle $\hat{x}_{i, i+1}$ aus, erhält man für die Erwartungsnutzen der benachbarten Verfahren $i$ und $i+1$ die Ausdrücke:

$$
\begin{aligned}
\left.E\left[U_{i}\right]\right|_{\widehat{x}=\widehat{x}_{i, i+1}} & =E\left[U\left(V_{0}-\widehat{K}+d_{i} \cdot e\right)\right] \\
\left.E\left[U_{i+1}\right]\right|_{\widehat{x}=\widehat{x}_{i, i+1}} & =E\left[U\left(V_{0}-\widehat{K}+d_{i+1} \cdot e\right)\right]
\end{aligned}
$$

Die Ausdrücke besitzen die gleiche Struktur wie (23) und (24) mit $a=V_{0}-\hat{K}$ und $b \in$ $\left\{d_{i}, d_{i+1}\right\}$. Da $d_{i+1}=p-c_{i+1}>d_{i}=p-c_{i}$, gilt jedoch für das Verhältnis der Risikoprämien der beiden Verfahren $\delta\left(d_{i+1}\right)>\delta\left(d_{i}\right)$, so dass $\left.E\left[U_{i}\right]\right|_{\hat{x}=\hat{x}_{i, i+1}}>\left.E\left[U_{i+1}\right]\right|_{\hat{x}=\hat{x}_{i, i+1}}$. Damit wird wie in Abschnitt $\mathrm{D}$ das kostengünstigere Verfahren $i+1$ durch das damit verbundene höhere Einkommensrisiko belastet, so dass sich der für den Verfahrensübergang relevante Schnittpunkt $\hat{x}_{i, i+1}^{*}$ gegenüber dem Schnittpunkt bei Risikoaversion $\hat{x}_{i, i+1}$ nach rechts verschiebt.

\section{Anmerkungen}

1 Vgl. dazu Luhmer (1992) sowie Schweitzer und Trossmann (1998), S. 76. ff.

2 Vgl. etwa Riebel (1994), S. 301 oder Fandel (2002).

3 Siehe etwa Jaedicke und Robichek (1964), Johnson/Simik (1971) oder Magee (1975). Zu nennen ist in diesem Zusammenhang auch die Arbeit von Adar, Barnea und Lev (1977), die zwar ein stochastisches Gewinnmaximierungsproblem bei Risikoaversion betrachten, aber keine Break-Even-Betrachtungen vornehmen.

4 Basierend auf der Analyse von Dillon und Nash (1978) wurde im deutschen Sprachraum von Schneider (1984) eine Diskussion über die Entscheidungsrelevanz von Fixkosten ausgelöst, vgl. dazu ausführlich Schweitzer/Küpper (2003), S. 472 ff. sowie Ewert/Wagenhofer (2006), S. $232 \mathrm{ff}$.

5 Ähnlich begründen auch Christensen/Demski (2003), S 104, die Verwendung exponentieller Nutzenfunktionen:..."we do not believe that wealth effects are a first-order effect when studying accounting theory".

6 Vgl. dazu Luhmer (1992).

7 Dabei unterstellen wir, dass der Erwartungswert $(\hat{x})$ im Verhältnis zur Varianz des Störterms $\left(\sigma^{2}\right)$ hinreichend gross ist, so dass die Wahrscheinlichkeit negativer Leistungsmengen gegen Null geht.

8 Vgl. etwa Bamberg und Coenenberg (2006), S. 108.

9 Vgl. dazu ausführlich Luhmer (1992), S. 556ff.

10 Vgl. etwa Horngren/Datar/Foster (2006), S. 71 f.

11 Für $F_{1}>0$ würde unterhalb der Break-Even-Menge $x_{1}=F_{1} / d_{1}$ nichts produziert werden. 
12 Für eine grundlegende Betrachtung des linearen Agency-Modells und dessen Grenzen siehe etwa Wagenhofer/Ewert (1993) und Hemmer (2004).

13 Die gewählte Form der Entlohnungsfunktion dient der Übersichtlichkeit der nachfolgenden Analyse. Abweichend könnte in Übereinstimmung mit dem Standard-LEN-Modell auch die Absatzmenge $x$ als Bemessungrundlage für die variable Entlohnung gewählt werden, ohne dass sich dadurch materiell andere Resultate ergeben würden.

14 Unter Verwendung der Anreizbedingung kann man den vorletzten Term in (18) auch schreiben als: $d_{i} \cdot a^{*}-a^{* 2} / 2-\mathrm{r} \cdot v^{2} \cdot d_{i}^{2} \cdot \sigma^{2}$.

15 Vgl. etwa Milgrom/Roberts (1992), S. 221.

16 Wäre der Nettobeitrag des Agenten nicht positiv, etwa weil dieser einen zu hohen Reservationsnutzen hat, würde ein rationaler Prinzipal auf die Einstellung des Agenten verzichten. Vgl. zu dieser Problematik Göx (2004).

17 Siehe dazu etwa Varian (1992), S. 185 f.

\section{Literatur}

Adar, Zvi/Barnea, Amir/Lev, Baruch (1977): A Comprehensive Cost-Volume-Profit Analysis under Uncertainty, The Accounting Review, Vol. 52, No.1, S. 137-149.

Bamberg, Günter/Coenenberg, Adolf G. (2006): Betriebswirtschaftliche Entscheidungslehre, 13. Auflage, Vahlen München.

Christensen, John A./Demski Joel S. (2003): Accounting Theory, McGraw-Hill, Boston et. al.

Dillon, Ray D./Nash, John F. (1978). The True Relevance of Relevance Cost, The Accounting Review, Vol. 53, No. 1, S. 11-17.

Ewert, Ralf/ Wagenhofer Alfred (2006), Interne Unternehmensrechnung, 6. Aufl. (2005), Springer Berlin.

Fandel, Günter (2002): Produktionscontrolling in: Küpper, Hans-Ulrich/Wagenhofer, Alfred (Hrsg.), Handwörterbuch Unternehmensrechnung und Controlling, 4. Aufl., SchäfferPoeschel-Verlag, Stuttgart, Sp. $1508-1521$.

Göx, Robert F. (2004): Erfolgsabhängige Gehälter, Belohnung für den Zufall und der Einfluss des Managements auf die Gestaltung seines eigenen Vergütungssystems, Schmalenbachs Zeitschrift für betriebswirtschaftliche Forschung, Sonderheft No. 51, 27-55.

Hemmer, Thomas (2004): Lessons Lost in Linearity: A Critical Assessment of the General Usefulness of LEN Models in Compensation Research, Journal of Management Accounting Research, Vol. 16, 149-162.

Horngren, Charles T./Datar, Srikant M./Foster, George (2006): Cost Accounting, International Edition, 12. Edition, Pearson/Prentice-Hall, Upper Saddle River.

Jaedicke, Robert K./Robichek, Alexander A. (1964): Cost-Volume-Profit Analysis under Conditions of Uncertainty, The Accounting Review, Vol. 39, No.4, S. 917-926.

Johnson, Glenn L./Simik II., S.Stephen (1971): Multiproduct C-V-P Analysis under Uncertainy, Journal of Accounting Research, Vol. 9, No.2, S.278-286.

Luhmer, Alfred (1992): Fixkosten-Controlling: ökonomische Grundlagen und Kalküle, in: Spremann, Klaus/ Zur, Eberhard: Controlling, Gabler Wiesbaden.

Magee, Robert P. (1975): Cost-Volume-Profit Analysis, Uncertainty and Capital Market Equilibrium, Journal of Accounting Research, Vol.13, No.2, S. 257-266.

Milgrom, Paul/Roberts, John (1992): Economics, Organization \& Management, International Edition, PrenticeHall, Englewood Cliffs.

Riebel, Paul (1994): Einzelkosten- und Deckungsbeitragsrechnung: Grundfragen einer markt- und entscheidungsorientierten Unternehmensrechnung, 7. Auflage, Wiesbaden.

Schneider, Dieter (1984): Entscheidungsrelevante fixe Kosten, Abschreibungen und Zinsen zur Substanzerhaltung. In: Der Betrieb (37), S. 2521-2528.

Schweitzer, Marcell/Tro1/4mann, Ernst (1998): Break-Even-Analysen: Methodik und Einsatz, 2. Aufl., Duncker\&Humblot, Berlin.

Schweitzer, Marcell/Küpper, Hans-Ulrich (2003): Systeme der Kosten- und Erlösrechnung, 8. Aufl., Vahlen München.

Varian, Hal R. (1992): Microeconomic Analysis, Third Edition, Norton, New York.

Wagenhofer, Alfred/Ewert, Ralf (1993): Linearität und Optimalität in ökonomischen AgencyModellen, Zeitschrift für Betriebswirtschaft, 63. Jg., S. 373-391. 


\section{Verfahrenswahl bei Risiko}

\section{Zusammenfassung}

Dieser Beitrag untersucht das Verfahrenswahlproblem bei Risiko. Wir zeigen, dass die unter Sicherheit abgeleiteten Entscheidungsregeln bei unsicherer Leistungsmenge nur dann gültig bleiben, wenn ein risikoneutraler Entscheider unterstellt wird. Bei Risikoaversion hängt die Lösung des Verfahrenswahlproblems von der unterstellten Zielgrösse ab. Bei kostenbasierter Betrachtungsweise erfolgt der Übergang auf Verfahren mit höheren Fixkosten und geringeren variablen Stückkosten früher als bei Sicherheit, bei deckungsbeitragsorientierter Betrachtungsweise dagegen später. Es kann auch vorkommen, dass Verfahren, die unter Risikoneutralität effizient sind, bei Risikoaversion von anderen Verfahren dominiert werden und umgekehrt. Dabei ist jedoch zu beachten, dass eine Verfahrenswahlentscheidung auf Basis einer isolierten Betrachtung des Kostenrisikos nur dann zulässig ist, wenn der Nutzen unabhängig von der Leistungsmenge ist. Schliesslich zeigen wir im Rahmen eines Agency-Modells, dass das Verfahrenswahlproblem trotz Risikoneutralität des Prinzipals nicht unabhängig von der Lösung des Anreizproblems gelöst werden kann, da andernfalls die Break-Even-Menge für den Verfahrensübergang überschätzt wird. Eine optimale Lösung des Verfahrenswahlproblems erfordert daher nicht nur eine Berücksichtigung der Risikoneigung des Entscheiders, sondern auch eine korrekte Berücksichtigung der übrigen Rahmenbedingungen des Entscheidungsproblems.

\section{Choice among different cost structures under risk}

\section{Summary}

This paper analyzes the choice among alternative fixed and variable cost structures under demand uncertainty. We show that the standard decision rules for the choice among cost structures under certainty continue to hold if the decision maker is risk neutral. If the decision maker is risk averse, the optimal cost structure depends on the decision model. With cost-based decision making, the break even quantities are lower than under certainty. If the decision is based on contribution margins, the opposite holds. That is, a cost structure with higher fixed and lower variable cost becomes attractive for a lower (higher) quantity than under certainty if the decision maker is risk averse and makes his decision on the basis of cost (contribution margin). We also show that cost structures that are dominated under certainty can become attractive for a risk averse decision maker. Finally, we provide a simple agency model and show that the choice among different cost structures can not be separated from the optimal solution of the agency problem even if the principal is risk neutral. More generally, our results suggest that a simple comparison of cost functions is usually not sufficient for an optimal choice between cost structures under uncertainty. 